\title{
Carbon Footprint Analysis in Metal Cutting Process
}

\author{
Qun Sun \\ School of Mechanical Engineering \\ Tongji University \\ Shanghai, China \\ sunqun0315@yahoo.com.cn
}

\author{
Weimin Zhang \\ Sino-German school \\ Tongji University \\ Shanghai, China \\ iamt@tongji.edu.cn
}

\begin{abstract}
Nowadays, with the greenhouse problem has been plaguing the world, the carbon footprint is also becoming an increasingly serious problem for manufacturing industry. To enable manufactures and operators of machining to take carbon footprint into their considerations in an objective way, this paper established the IPO model based on the analysis of the source of carbon footprint in metal cutting process. It aims to focus on environmental sustainable aspect, so as to seek for the optimal planning for reducing environmental impact in machining process.
\end{abstract}

\section{Keywords- Carbon Footprint; metal cutting process; IPO model}

\section{INTRODUCTION}

With the warming trend has accelerated as well as resource prices rise, the human community is facing a serious challenge to sustainable development, greenhouse gases, energy and resource conservation has become the most popular topics. Up till now, the manufacturer's power source still comes from coal as raw material of carbon-based resources (such as coal, oil ,gas, and so on) at present, the resulting gas emissions accounted for more than half of the total global greenhouse gas emissions [1], that is, the carbon emissions are the most important factors affecting global warming.

In machinery manufacturing, metal cutting process has the highest unit energy consumption. Take typical processes such as milling, turning and other metal cutting for example, unit energy consumption account for $66 \sim 82 \mathrm{MJ} / \mathrm{kg}$ in numerical control machining process, $50 \%$ higher than the consumption by using the forming methods such as forging, casting and so on [2].

Furthermore, the energy consumption of coarse processing is about $60 \%$ of the total energy consumption, finishing even reached 95\% [3]. Obviously, research of the resource consumption, energy conservation and emissions reduction for the metal cutting process has enormous potential and ecological urgency.

Due to the recent requirement of the environmental protection, carbon footprint is the focus topic in many research fields recently. In this paper, carbon footprint is taken as the unify index which including important factors in metal cutting process. Based on the boundary set for calculation, the source of greenhouse gas emissions is analyzed in detail, then, the IPO model is established for calculation.

\section{CARBON FOOTPRINT INTRODUCTION}

\section{A. Concept introduction}

A carbon footprint has historically been defined as "the total set of greenhouse gas (GHG) emissions caused by an organization, event, product or person [4]."

With global warming increasingly causes governments and widespread public concern, carbon footprint analysis method has recently become one of the hot research topics in academic. In general, carbon footprint is calculated based on the climatechange impact of emissions from the six types of the Kyoto Protocol greenhouse gases. These greenhouse gases include carbon dioxide $\left(\mathrm{CO}_{2}\right)$, methane $\left(\mathrm{CH}_{4}\right)$, nitrous oxide $\left(\mathrm{N}_{2} \mathrm{O}\right)$, hydrofluorocarbons (HFCs), perfluorocarbons (PFCs), and sulfur hexafluoride (SF6)[5].

A measure of the total amount of carbon dioxide (CO2) and methane (CH4) emissions of a defined population, system or activity, considering all relevant sources, sinks and storage within the spatial and temporal boundary of the population, system or activity of interest. The more the amount of carbon dioxide generated, the greater the carbon footprint, and vice versa.

\section{B. Generate calculate method}

For simplicity of reporting, it is often expressed in terms of the amount of carbon dioxide, or its equivalent of other emitted GHGs, it is calculated as carbon dioxide equivalent (CO2e) using the relevant 100-year global warming potential (GWP100)."[5]

Carbon footprint is expressed in carbon dioxide-equivalent (CO2e) which is obtained by multiplying the mass emissions of each GHG by its Global Warming Potential (GWP). GWP is the ability of each greenhouse gas to trap heat in the atmosphere relative to carbon dioxide, which serves as the reference gas [6]. Table 1 lists the six Kyoto Protocol GHGs and their GWP factors from IPCC [5].

Due to greenhouse gas emissions include direct emissions, indirect emissions and other emissions; it can also be calculated based on the activity data, emission factors of the use of energy, resources, and greenhouse gas emissions, formulated as follows:

$$
E_{C O 2 e}=M_{i} \times G W P_{i}=E F_{i} \times \sum_{j} A_{j}^{T}
$$


Where $E_{C O 2 e}$ is the total $\mathrm{CO}_{2}$-eq emissions ; $M_{i}$ is the quantity of greenhouse gas $i$; $G W P_{i}$ is its Global Warming Potential ability of each greenhouse gas; $E F_{i}$ is the ith greenhouse gas emission; $i=\left(\mathrm{CO}_{2}, \mathrm{CH}_{4}, \mathrm{~N}_{2} \mathrm{O}, \ldots\right) ; A_{j}^{T}$ is the activity data at the process step $j$.

TABLE I. THE SIX KYOTO PROTOCOL GASES AND THEIR GWP FACTORS[5]

\begin{tabular}{ll}
\hline Greenhouse Gas & GWP (100 years) \\
\hline Carbon dioxide (CO2) & 1 \\
Methane (CH4) & 25 \\
Nitrous oxide (N2O) & 298 \\
Hydrofluorocarbons (HFCs) & $124 \sim 14,800$ \\
Perfluorocarbons (PFCs) & $7,390 \sim 12,200$ \\
Sulfur hexafluoride (SF6) & 22,800 \\
\hline
\end{tabular}

\section{CARBON FOOTPRINT ANALYSIS FOR THE METAL} CUTTING PROCESS

Metal cutting processes are industry processes in which metal parts are shaped by material removal processes involving the interaction between the cutting tool, the material to be cut, and the cutting fluid [7]. These three elements are main parts of machining activity, they are including the material and energy consumption with relationship to carbon footprint not only in terms of cost and competitiveness, but also in environmental aspect.

\section{A. carbon footprint boundary set based on the processing technic}

Definition of boundaries set is the premise for carbon footprint calculation, it plays an important role in carbon footprint calculation, meanwhile, there will be different depending on the research object and perspective. It has been defined in GHG Protocol and PAS 2050 in detail, two international standards both took it as an important part of the carbon footprint analysis. Based on GHG Protocol standards, the paper takes the metal machining system as the subject. Take products' machining process for example, after raw materials in the workshop are transmitted to the various procedures, for one single step process, take the single technic process as the boundary for the analysis of the production carbon footprint. Similarly, with the entire production lines for the boundary when calculate the carbon footprint in the whole machinery processing.

In essence, the boundary set analysis is the purpose of the research: to estimate and analysis of machining process of greenhouse gas emissions, hence to select the appropriate means of production process technic or low emission materials to identify potential to reduce carbon footprint. Therefore, boundary limits involving all important material and energy flow of the stream of input and output. Within this boundary set, carbon dioxide equivalent emissions related to identification and classification of activities can be divided into the process of direct, indirect and optional greenhouse gas emission [8].

\section{B. GHG emission source analysis based on boundary set}

From what has been said, greenhouse gas emissions include direct emission, indirect emission and optional emission. Direct emission, mainly refers to the emissions generated by the processing activities in boilers, furnaces, vehicle transport produce combustion emissions, as well as the production of chemical process equipment. Indirect emission mainly refer to corporate greenhouse gas emissions generated by power consumption, including numerical control machine tools, hydraulic device, transmission device and other electric devices. Besides, optional emission is the emission for all components from the original material refining, processing, transport, transport fuel emissions, and waste materials recovery after all the activities in the greenhouse gas emissions (see Figure 1).

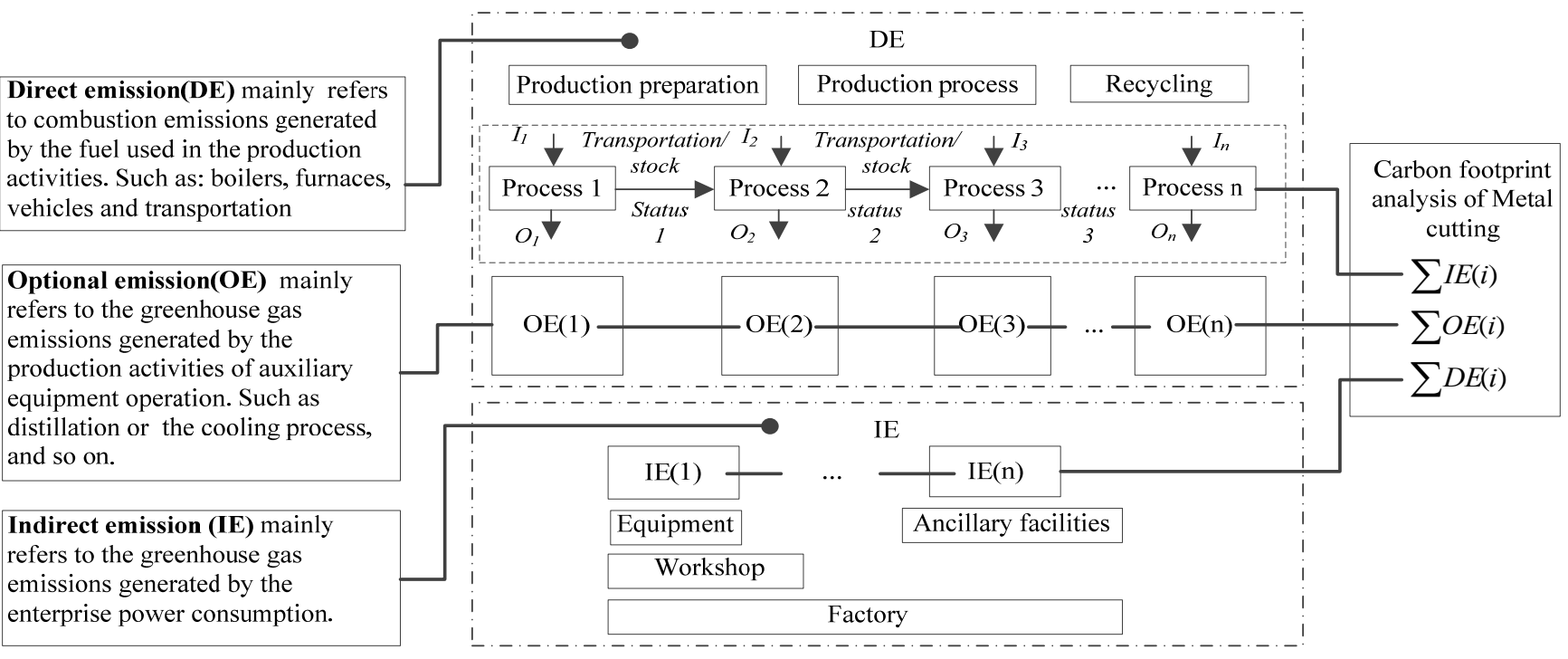

Figure 1. The analysis of the source of carbon footprint in machining process 
As a result of product cycle involved in optional emissions is too long, even some scholars put forward the LCA method can be calculated it theoretically, while the calculation is very complex. It is notable that, the theory was put forward in this paper is especially for the former two emissions condition.

\section{IPO FUNCTION MODEL FOR METAL CUTTING PROCESS}

Production process is the whole process of transforming raw materials into finished products, can also be seen as a resource-environment input -output processes. Based on the manufacturing process chain and IPO (IPO, input-process- output) graph, complex manufacturing processes can be decomposed into a series of process unit, establish IPO correlation function model on the basis of the analysis of resource consumption and environmental impact.

Due to the various indicators and environments for production process, the unify index can behalf the resources consumption and ecological influences is necessary for the expression and evaluation.

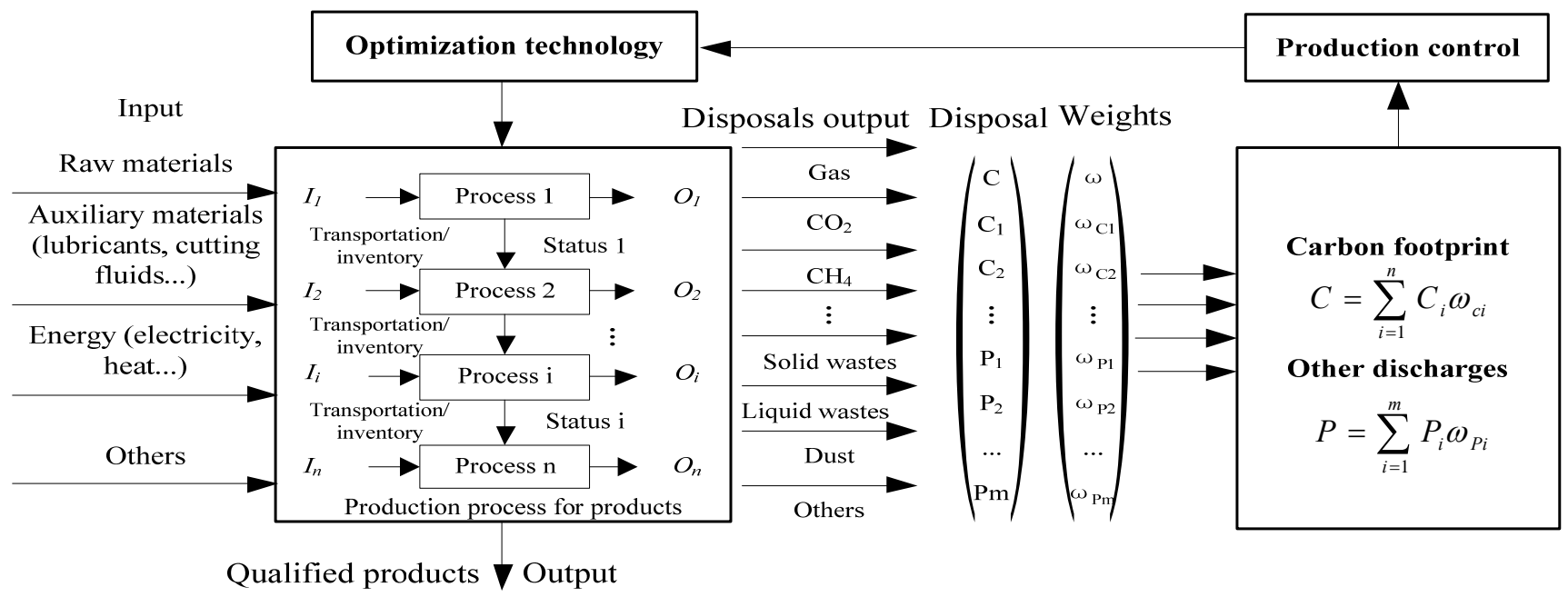

Figure 2. IPO function model for production process

According to the analysis results recently [9,10,11], carbon footprint is the index that describes the main consumption and its ecological influences for production process. On the one hand, carbon footprint is the one index which can calculate the main consumption for production process, such as consumption of material, electricity, cutting tool, cutting fluid, coolant, lubricant oil and so on. On the other hand, it is also the ecological index can reflect the impacts for production process from an ecological perspective. For the main gas emissions in production are some greenhouse gases, such as $\mathrm{CO}_{2}, \mathrm{CH}_{4}$ and $\mathrm{N}_{2} \mathrm{O}$, among them, $\mathrm{CO}_{2}$ is the dominant environmental burden in the machining operation concerning global warming by comparing $\mathrm{CO}_{2}$ emission with the equivalent $\mathrm{CO}_{2}$ emission of $\mathrm{CH}_{4}$ and $\mathrm{N}_{2} \mathrm{O}$ [12]. A measure of the total amount of carbon dioxide $\left(\mathrm{CO}_{2}\right)$ and other greenhouse gases is calculated as carbon dioxide equivalent $\left(\mathrm{CO}_{2} \mathrm{e}\right)$ by different emission factors. Therefore, it is reasonable to take carbon footprint as the unify index to evaluate and control the production process is well done or not, which can represent the resource consumption, most of the waste gas emission from manufacturing processes, as well as some of the other waste disposal (see Figure 2).

There are many indicators relatives to carbon discharge, assuming there are $\mathrm{n}$ kinds of processes relatives to carbon footprint during the whole production process. The calculation formula is given by:

$$
C=\sum_{i=1}^{n} C_{i} \omega_{c i}=C_{1} \omega_{c 1}+C_{2} \omega_{c 2}+\ldots+C_{i} \omega_{c i}+\ldots+C_{n} \omega_{c n}
$$

Where $C_{i}(i=1,2, \ldots, n)$ is the activity data, the optional $\mathrm{CO}_{2} \mathrm{e}$ emissions induced from the use of materials/chemicals in an assessed process; $\omega_{C i}$ the emission factor of the $n t h$ type of material.

According to the principles of resource and environmental properties (closet physical characteristics, environmental characteristics, and so on), divided other waste disposals that unrelated to carbon footprint into $m$ kinds. The optional other emissions unrelated carbon footprint from the use of materials and chemicals can be accounted by:

$$
\begin{gathered}
P=\sum_{i=1}^{m} P_{i} \omega_{P i}=P_{1} \omega_{P 1}+P_{2} \omega_{P 2}+\ldots+P_{i} \omega_{P i}+\ldots+P_{m} \omega_{P m} \\
\left(\omega_{C 1}+\omega_{C 2}+\ldots+\omega_{C n}\right)+\left(\omega_{P 1}+\omega_{P 2}+\ldots+\omega_{P m}\right)=1
\end{gathered}
$$

Where $P_{i}(i=1,2, \ldots, m)$ is also the activity data, the discharge unrelated to carbon footprint(such as noise, dusts, etc.), $\omega_{P i}$ the emission factor of the $m t h$ type of material.

From the formula what has been proposed, it can be seen that it is impossible to calculate the carbon footprint for a metal cutting technic, hence to select the best process chain or ensure minimum GHG emissions without having detailed previous knowledge of process and machine. In general, the activity data can usually collected from real processes and empirical research. While the exact calculation depends on the activity data of the classified material and its emission factor relatives to carbon footprint, it is important to note that the factor must also be appropriately accurately collected. 
Contrary to the acquisition of activity data, the compilation of emission factors is more complicated for collection [7]. Some authorities such as EPA, IPCC (Intergovernmental Panel on Climate Change), EU (European Union) and many countries maintain compilations of emission factors that can be used in various emission evaluation, while, they are still not readily available at present. According to EPA (United States Environmental Protection Agency), an emission factor is a representative value that attempts to relate the quantity of a pollutant released to the atmosphere with an activity associated with the release of that pollutant [13].

\section{SUMMARY AND OUTLOOK}

In this paper, based on the machining characteristics, the boundary set and source for carbon footprint is put forward in detail. Furthermore, a modeling framework for carbon footprint forecasting in metal cutting process has been presented. For the purpose is to provide the way for production process optimization essentially. Future work will be directed towards extending this methodology, optimization and merging with optimal control simulation to provide convenient tools for manufacturing industries.

\section{ACKNOWLEDGEMENTS}

This work is supported by the Key Projects in the National Science \& Technology Pillar Program during the Twelfth Fiveyear Plan Period (2012BAF01B02) "Key technologies development and application demonstration for high speed CNC machine tool of green manufacturing." The authors would like to thank all the members of the projects.

\section{REFERENCES}

[1] World Resources Institute. (2005) World Greenhouse Gas Emissions: 2005. Available at: http://www.wri.org/chart/world-greenhouse-gasemissions-2005 [Accessed 10 August 2009].

[2] A. Herbert Fritz, Günter Schulze, G. Fertigungstechnik. neu bearbeitete Auflage. Springer Verlag. 2010.

[3] Fertigungstechnisches Kolloquium Stuttgart 2010. Stuttgart. 2010.

[4] S.Thurwachter, D.J Bauer, P.S Sheng, "Integration of environmental factors in surface planning: Part2-Multi-criteria hazard control, transactions of NAMRI”, 5th ed., vol 26, 1998, pp. 115 -121.

[5] Intergovernmental Panel on Climate Change (IPCC), Climate change 2007: The physical science basis. Contribution of working group I to the fourth assessment report, Solomon SD and Qin M (eds.), Geneva, Switzerland: IPCC, 2007.

[6] Inventory of U.S. greenhouse gas emissions and sinks1990-1994, Washington D.C.,Nov.1995.

[7] Oliver Avram, Ian Stroud, Paul Xirouchakis, “A multi-criteria decision method for sustainability assessment of the use phase of machine tool systems”, Int J Adv Manuf Technol, 2011, pp. 811-828.

[8] Qizhen Yang, Zhiqi Shen, "Modeling and scenarios analysis of environmental sustainability in waste management," International Journal of Information Technology, 2nd ed., vol. 16, 2010.

[9] S. Tridech, K. Cheng, "Low carbon manufacturing: characterization, theoretical models and implementation," International Journal of Manufacturing Research, 2nd ed.,vol. 6, 2011, pp.110-121.

[10] T. Wiedmann, J. Minx, "Ecological economics research trends”, edited by Carolyn C. Pertsova Publications, Hauppauge, 2008.

[11] A. Laurent, S.I. Olsen, M.Z. Hauschild, "Carbon footprint as environmental performance indicator for the manufacturing industry”, CIRP Annals - Manufacturing Technology, vol 59, 2010, pp. 37-40.

[12] Hirohisa Narita,Nelfa Desmira,Hideo Fujimoto, "Environmental burden analysis for machining operation using LCA method,” Manufacturing Systems and Technologies for the New Frontier, Part 2, 2008, pp. 65-68.

[13] U.S.Environmental Protection Agency (EPA), Emissions factors and AP-42, available at http://www.epa.gov/ttn/chief/ap42/index.html (Accessed in Dec 2010). 